\title{
The effect of posture on the normal and pathological auditory startle reflex
}

\author{
P Brown, B L Day, J C Rothwell, P D Thompson, C D Marsden
}

\begin{abstract}
The effect of posture on the EMG pattern of the normal auditory startle reflex was investigated. The startle response to an unexpected auditory tone was studied in eleven normal subjects when standing, and in six normal subjects when sitting relaxed or tonically plantar flexing both feet. Reflex EMG activity was recorded in the tibialis anterior and soleus about twice as frequently when standing, than when sitting relaxed. In addition, the median latencies to onset of reflex EMG activity in the tibialis anterior and soleus were about 40 and 60 ms shorter during standing, than when sitting relaxed. No short latency EMG activity was recorded in the calf muscles during tonic plantar flexion of the feet; while sitting. The effect of posture on the EMG pattern of the pathological auditory startle reflex was studied in five patients with hyperekplexia. In three patients the latency to onset of reflex EMG activity in the tibialis anterior was shorter when standing, than when sitting relaxed. The EMG pattern of the reflex response to sound was studied in detail in two of these patients and consisted of up to three successive components. The expression of each EMG component depended on the postural set of the limbs. In particular, a distinct short latency component was found in posturally important muscles following auditory stimulation. This short latency component was not recorded when sitting relaxed. It is concluded that the EMG pattern of the physiological and pathological auditory startle response is not fixed, but may change with the postural stance of the body. This finding supports the theory that the normal startle reflex and the abnormal startle reflex in hyperekplexia have a common brainstem origin.
\end{abstract}

We have recently defined the electromyographic pattern of the normal auditory startle response in humans. ${ }^{1}$ The order of recruitment of muscles suggests that the activity responsible for the startle response originates in the caudal brainstem, and is conducted up the brainstem and down the spinal cord by relatively slowly conducting efferent pathways. We have also investigated the electromyographic pattern of the abnormal startle response in certain patients with familial and acquired startle disorders. ${ }^{2}$ In these patients we have shown that the pattern of muscle recruitment in the abnormal startle response is very similar to that in the physiological auditory startle response, and may therefore represent activity in the same brainstem efferent system. We have used the term hyperekplexia to describe those patients with a startle syndrome due, pathophysiologically, to an exaggerated normal startle reflex. ${ }^{2}$

The exact functional importance of the startle reflex to humans remains unclear. Landis and $\mathrm{Hunt}^{3}$ stressed the relative immutability of the startle response, despite changes in posture and activity, and as a result the auditory startle reflex has been considered little more than a basic and stereotyped alerting response. ${ }^{4}$

There is, however, some evidence to suggest that the auditory startle reflex in humans may be more than a simple stereotyped flexion reflex. EMG activity may be recorded in extensor muscles during the startle reflex, ${ }^{1}$ and Rossignol ${ }^{5}$ found that the activity recorded in extensor muscles increased during or after tonic extension of the relevant joint.

We show that the electromyographic pattern of the normal and abnormal auditory startle reflex may vary with posture. We suggest therefore that the physiological importance of the auditory startle reflex in humans lies in the rapid accomplishement of a defensive stance with maximum postural stability.

\section{Subjects}

The response to auditory stimulation was investigated in a total of eleven healthy subjects (mean age 30 years, range 18 to 47 years old), and five patients with hyperekplexia and a pathologically exaggerated startle reflex. The diagnoses in the five patients were hereditary hyperkplexia in cases 1 and 3, postanoxic encephalopathy in case 2 , probable multiple sclerosis in case 4, and encephalomyelitis with rigidity, the "Jerking stiff person syndrome" reported by Leight $e t$ al in case 5 . Their clinical details and basic neurophysiological findings have been described previously (reference 2, cases $1,8,3,5$ and 7 respectively).

Both the normal subjects and the patients gave their informed consent to the electrophysiological studies.

\section{Methods}

Electromyogram (EMG) recordings were 
made using bipolar silver/silver chloride electrodes placed $2 \mathrm{~cm}$ apart longitudinally over the muscle bellies. Magnetic stimulation of the cortex was performed with a $9 \mathrm{~cm}$ diameter circular stimulator coil using a Novametrix Magstim 200. Auditory startle responses were recorded by triggering the computer at the time of delivery of an auditory tone burst of $1000 \mathrm{~Hz}$ frequency, $50 \mathrm{~ms}$ duration and $124 \mathrm{~dB}$ presented binaurally through earphones. The sampling rate was $2000 \mathrm{~Hz}$ per channel. The latency to onset of reflex EMG activity was measured by visual inspection of the computer display of unrectified records of single trials. The latency of the initial voltage sustained above the background level of EMG activity was taken to be the start of reflex EMG activity.

In experiments on healthy subjects the reflex EMG activity in the sternocleidomastoid, tibialis anterior and soleus was recorded to an unexpected auditory tone repeated randomly about every 15 minutes. Eleven subjects were tested while standing with their feet close together. Reflex EMG activity was recorded in the calf muscles of eight of the subjects on standing, and of these, two subjects were tested while sitting relaxed, two while sitting tonically plantar flexing both feet, and four subjects were tested under all three conditions. Subjects kept their eyes closed during each condition. Four to six trials were recorded under each condition, usually over more than one session. The order of conditions was varied between subjects.

In the experiments on patients with hyperekplexia the auditory tone was repeated randomly about every three minutes.

Medians and ranges were recorded. Statis-

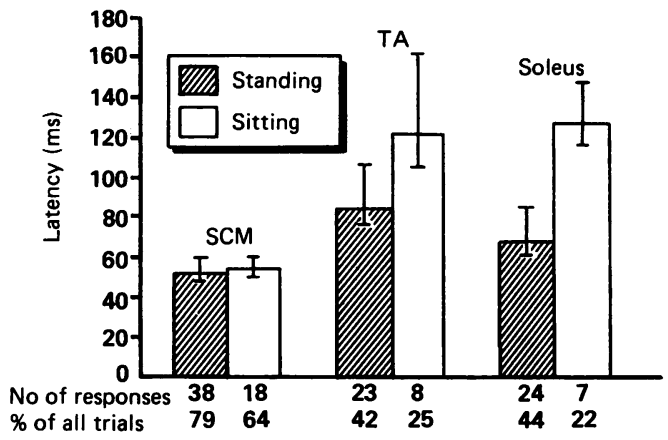

Figure 1 The effect of sitting and standing on the pattern of EMG activity in the normal auditory startle response. The median and interquartile range of the latency to onset of reflex EMG activity elicited by an unexpected auditory stimulus (a 50 ms duration tone of $1000 \mathrm{~Hz}$ and $124 \mathrm{~dB}$ ) is shown for eleven healthy subjects when standing, and six healthy subjects when sitting relaxed. There was no significant difference in the latency to onset of reflex EMG activity in sternocleidomastoid (SCM) between sitting and standing. There was, however, a significant difference in latency to onset of EMG activity between standing and sitting in tibialis anterior $(T A)$ and soleus $(p=0.0051$ and 0.0002 respectively). Reflex EMG activity in the various muscles was recorded in only some trials. The number of trials in which reflex EMG activity was recorded in each muscle, and the percentage of the total number of trials in which responses were present in each muscle, are shown at the bottom of the figure. Reflex EMG activity to unexpected auditory stimulation was recorded in tibialis anterior and soleus more frequently when standing, than when sitting. tical analysis was performed using the MannWhitney U test.

\section{Results}

THE EFFECT OF STANDING ON THE AUDITORY STARTLE RESPONSE IN NORMAL SUBJECTS

The auditory startle response was recorded in eleven subjects, while standing, and in six of these subjects, while sitting relaxed. Reflex EMG activity was recorded in the calf muscles, in at least one trial, in eight subjects, while standing, and three subjects, while sitting relaxed. Figure 1 shows the median latencies to onset of reflex EMG activity in sternocleidomastoid, tibialis anterior and soleus, and the percentage of the total number of trials in which reflex EMG activity was recorded in each muscle, following auditory stimulation while standing and sitting.

The percentage of trials in which reflex EMG activity was recorded in sternocleidomastoid, and the latency to onset of reflex EMG activity in this muscle did not differ between standing and sitting. In contrast, reflex EMG activity was recorded in the tibialis anterior and soleus about twice as frequently when standing, than when sitting relaxed. In addition, the median latencies to onset of reflex EMG activity in tibialis anterior and soleus were about 40 and $60 \mathrm{~ms}$ shorter during standing, than when sitting relaxed. The difference in latency to onset of EMG activity in tibialis anterior and soleus between standing and sitting was significant ( $\mathrm{p}=0.0051$ and 0.0002 respectively).

Standing involved both a change in postural set and an increase in the level of background EMG activity in the lower limbs. (There was no change in the level of background EMG activity recorded in sternocleidomastoid between sitting and standing, and it is likely that the muscles of the neck were posturally important during both sitting, with the head unsupported, and standing). An attempt was made to control for the increase in background EMG activity in the lower limbs by asking six of the subjects to tonically plantar flex both ankles while sitting, by pushing their toes into the floor. Under this condition there was, again, no significant change in the percentage of trials in which reflex EMG activity was recorded in sternocleidomastoid $(58 \%)$, or in the median latency to onset of reflex EMG activity in this muscle ( $55 \mathrm{~ms}$, range 44 to $76.6 \mathrm{~ms}$ ). However, reflex EMG activity was recorded in the calf muscles in only three out of 23 trials under this condition, and this was of long latency (range 173 to $191 \mathrm{~ms}$ ). Figure 2 illustrates the effect of unexpected auditory stimulation in a normal subject while standing, sitting relaxed and sitting tonically plantar flexing both ankles.

\section{THE EFFECT OF STANDING ON THE} PATHOLOGICAL AUDITORY STARTLE RESPONSE The effect of standing on the abnormal startle response was investigated in five patients with hyperekplexia. Figure 3 compares the latency to onset of reflex EMG activity in the tibialis anterior while sitting and standing. When sitting the latency to onset of the reflex EMG 


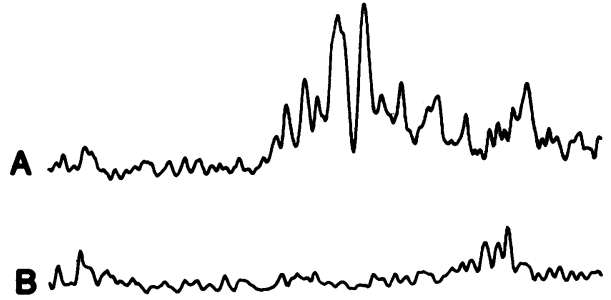

C

Figure 2 The EMG response to auditory stimulation in the normal subject when standing. Record $2 A$ shows the short latency reflex EMG activity in soleus elicited by unexpected auditory stimulation in a normal subject when standing. Short latency reflex EMG activity was absent when sitting, while tonically plantar flexing both ankles $(2 B)$, or when sitting relaxed (2C). Each record consists of the average of the rectified EMG activity in five trials. $A 124 d B$ tone of $50 \mathrm{~ms}$ duration was given at the start of each trial. The horizontal and vertical calibration lines represent $20 \mathrm{~ms}$ and $0.1 \mathrm{mV}$ respectively.

response in the tibialis anterior was short (about $85 \mathrm{~ms}$ ) and similar to that recorded in normal subjects when standing. When cases 3 , 4 , and 5 were standing the latency to onset of reflex EMG activity in the tibialis anterior shortened significantly still further $(p=0.05$ in each case). In cases 1 and 2 there was no change in latency while standing.

The response to auditory stimulation in cases 1, 2 and 3 consisted of a single uninterrupted burst of EMG activity in the trunk and limb muscles of 100 to $400 \mathrm{~ms}$ in duration, whether standing or sitting. In cases 4 and 5, however, the response to auditory stimulation consisted of several clearly definable components of EMG activity. The change in these EMG components with different postures, and at rest, was studied in more detail.

THE EFFECT OF POSTURE ON THE PATHOLOGICAL AUDITORY STARTLE REFLEX IN CASES 4 AND 5 The EMG pattern of the reflex response to auditory stimulation was investigated in cases 4 and 5 while sitting relaxed, standing unsupported, and sitting tonically plantar flexing both ankles. Examples of the reflex EMG responses recorded under the three different test conditions are shown in figs 4 and 5 . Up to three clearly definable successive components were present in the reflex EMG response, under the different test conditions. These three components will be termed A, B and C. The median latencies of each component are shown for representative muscles in the table.

The effects of the different test conditions differed between the three components. When sitting relaxed (top records in figs 4 and 5), the reflex EMG response to auditory stimulation in both patients consisted of two separate successive components, $B$ and $C$. When standing (middle records in figs 4 and 5) a third component of very early latency was recorded in trunk and lower, but not upper, limb muscles. In addition, although the $B$ component remained on standing, the $\mathrm{C}$ component became much smaller or disappeared.

The reflex response to auditory stimulation was also investigated as cases 4 and 5 sat pushing their feet into the ground. In this situation (bottom records in figs 4 and 5) the level of background EMG activity recorded in the trunk and lower limb muscles equalled or exceeded that recorded in these muscles on standing. Despite this, the effect of tonic activity when sitting was intermediate between the effect of sitting relaxed and that of standing. Thus the A component was visible, but small, and the $\mathrm{B}$ and $\mathrm{C}$ components were almost the same as when sitting relaxed.

In a further set of experiments the upper limbs were made posturally important to see whether this would bring out an early latency $A$ component in the arm muscles. Case 4 was asked to crouch on his hands and knees, with his centre of balance slightly forwards. Case 5 was asked to stand on a soft pillow, with her eyes shut, while steadying herself by holding on to a fixed support with the right hand. In both patients, an A component appeared in the reflex response to auditory stimulation in the biceps, triceps, and the forearm flexors and extensors (The intrinsic hand muscles were not recorded from during this experiment). The B component remained in the arm muscles whether the upper limb was used posturally or not. The C component was diminished in size or absent. The bottom biceps record in fig 4 shows the effect of crouching on all fours upon the pattern of reflex EMG activity in case 4. The early latency $A$ component did not appear in the reflex EMG response to sound recorded in the upper limbs when case 5 was asked to make a tight fist with the right hand, while standing normally.

In summary, the A component was recorded when a muscle was posturally active, and to a lesser extent when the muscle was tonically

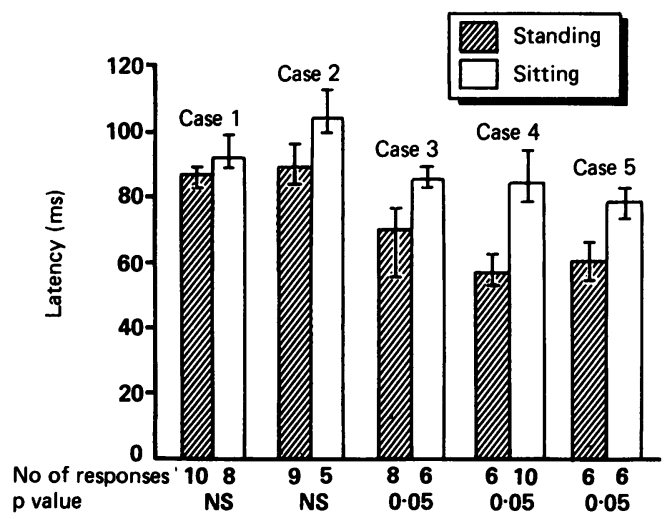

Figure 3 The effect of sitting and standing on the latency to onset of reflex EMG activity in the tibialis anterior in the abnormal auditory startle response. The median and interquartile range of the latency to onset of reflex EMG activity in tibialis anterior is shown for five patients with hyperekplexia, while standing and sitting. There was a significant difference in the latency to onset of reflex EMG activity in tibialis anterior between standing and sitting in cases 3, 4 and 5. The number of trials in which reflex EMG activity in tibialis anterior was recorded in each patient is shown at the bottom of the figure. The auditory stimulus was a $50 \mathrm{~ms}$ duration tone of $1000 \mathrm{~Hz}$ and 124 dB. 


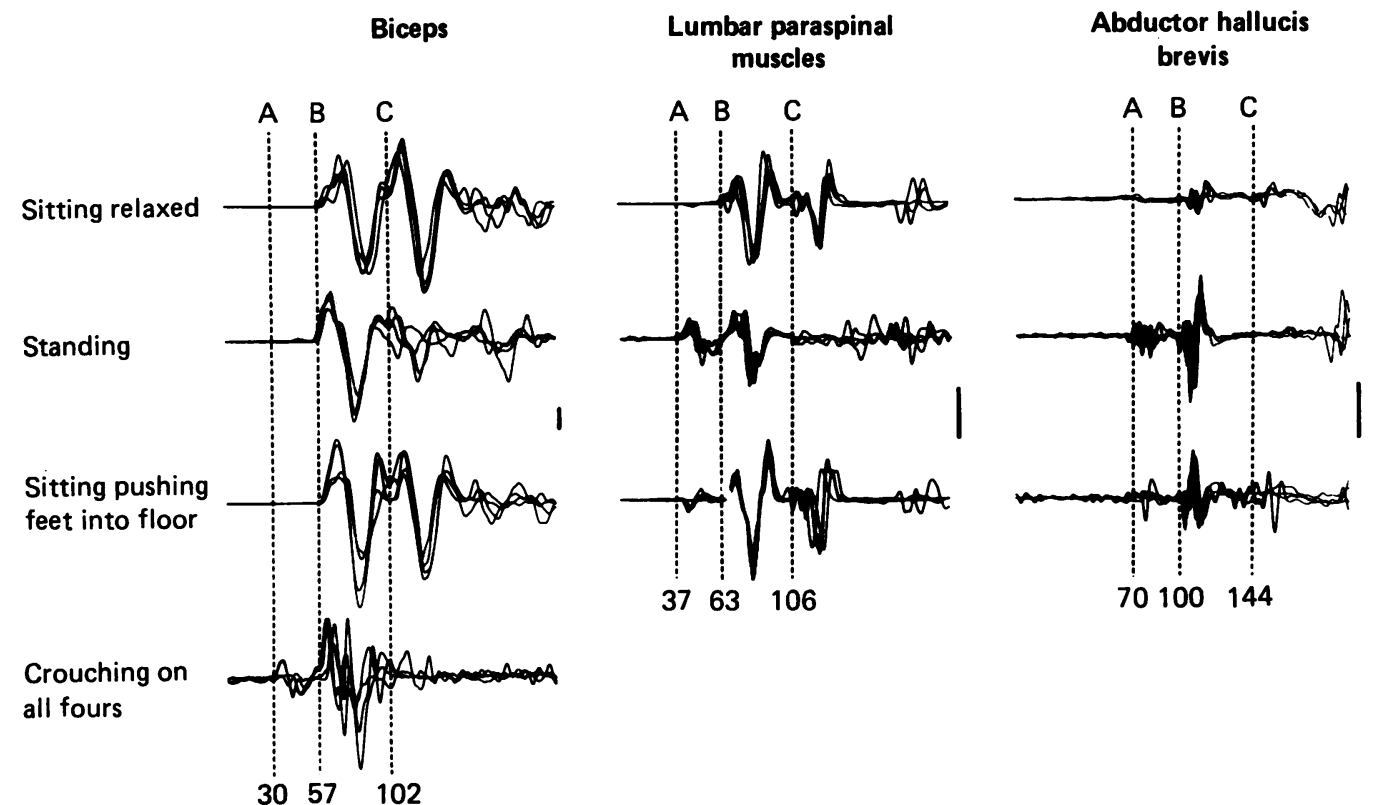

Figure 4 The EMG pattern of the reflex response to auditory stimulation in the biceps brachii, lumbar paraspinal muscles and abductor hallucis brevis in case 4 . The upper three records show the reflex EMG activity in response to auditory stimulation in each muscle when sitting, relaxed. This consists of two major components marked $B$ and $C$ respectively. The middle three records show the reflex EMG activity when standing. A large short latency $A$ component is seen, preceding the $B$ component in the lumbar paraspinal muscles and abductor hallucis brevis. The $C$ component is greatly diminished in biceps brachii and has disappeared in the lumbar paraspinal muscles and abductor hallucis brevis. The lower three records show the reflex EMG activity in response to auditory stimulation when sitting, pushing both feet into the ground. A small short latency $A$ component is visible in the lumbar paraspinal muscles and abductor hallucis brevis. The $B$ and $C$ components are relatively unchanged in all three muscles, compared to sitting relaxed. The differences between the reflex EMG patterns on standing and sitting, pushing the feet into the floor, occur despite comparable levels of background EMG activity. The bottom left record shows the reflex EMG activity in biceps brachii in response to auditory stimulation when crouching on all fours, with the centre of balance shifted slightly forwards. $A$ short latency $A$ component is now seen, preceding the $B$ component in biceps brachii. The $C$ component is absent. Each record consists of four superimposed single trials of unrectified EMG activity. The auditory stimulus, a 50 ms duration tone of $1000 \mathrm{~Hz}$ and $124 \mathrm{~dB}$, was delivered at the beginning of each trial. The total sweep duration of each trial was $200 \mathrm{~ms}$. Each vertical calibration bar represents $1 \mathrm{mV}$.

activated. The A component was not recorded when sitting relaxed. The $B$ component remained unchanged whether the patient was stitting or standing. The $\mathrm{C}$ component was recorded when sitting, but was diminished or absent on standing or during postural use of the arm. It was not affected by tonic activation of the muscle.

All three reflex components in cases 4 and 5 were characterised by a difference in latency to onset of EMG activity between the biceps and tibialis anterior, which exceeded that recorded in the same patient following magnetic stimulation of the motor cortex (table). In addition, in case 4 , the differences in latency to onset of EMG activity between the biceps and the intrinsic hand muscles for components $B$ and $C$, and between the tibialis anterior and the intrinsic foot muscles for components $A, B$ and $C$ were very long, compared with the differences in latency following magnetic stimulation of the motor cortex (table).

\section{Discussion}

THE EFFECT OF POSTURE ON THE NORMAL AUDITORY STARTLE REFLEX

In a recent investigation of the normal auditory startle response, ${ }^{1}$ we found that the reflex EMG activity elicited by unexpected auditory stimulation in normal subjects who were sitting was infrequently recorded in the muscles of the lower limbs, and when recorded, was of relatively long latency (in excess of $100 \mathrm{~ms}$ ). Here we have shown that when normal subjects are standing, reflex EMG activity is recorded in the muscles of the lower limbs about twice as frequently as when sitting, and that such reflex EMG activity is of much shorter latency, than when sitting. These changes are the consequence of the change in postural set of the lower limbs on standing, and not due to the increased level of background EMG activity present during standing. Unexpected auditory stimulation during tonic plantar flexion of both feet in the sitting subject did not shorten the latency of the reflex response recorded in the calf muscles, and did not increase the frequency of such responses.

THE EFFECT OF POSTURE ON THE ABNORMAL AUDITORY STARTLE REFLEX

The effect of standing on the abnormal startle response was also studied. In three out of five patients with familial or symptomatic hyperekplexia there was a significant shortening of reflex latency in the tibialis anterior while standing. This effect of posture on the pattern of EMG activity in the abnormal startle reflex was studied in detail in cases 4 and 5 . In these two patients the reflex response to sound consisted of up to three successive components (A, B and C) of EMG activity. The expression of the three EMG components in the reflex response 


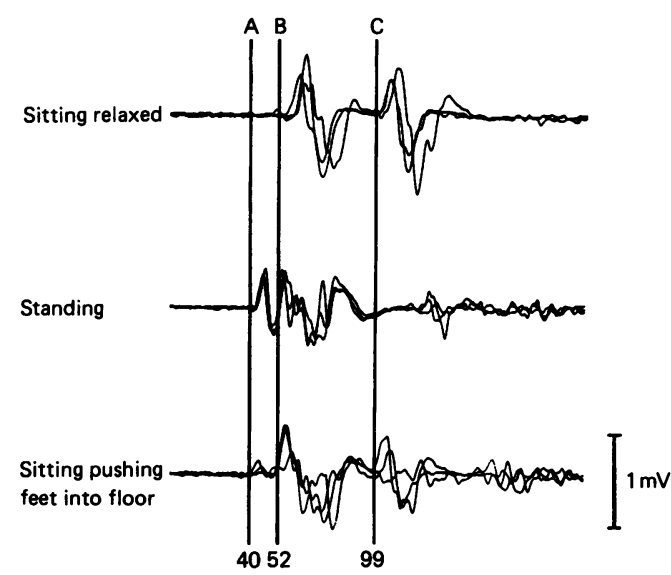

Figure 5 The EMG pattern of the reflex response to auditory stimulation in the lumbar paraspinal muscles in case 5. The upper record shows the reflex EMG activity in response to auditory stimulation when sitting, relaxed. This consists of two major components marked $B$ and $C$ respectively. The middle record illustrates the reflex EMG activity when standing. $A$ large short latency $A$ component is seen, preceding the $B$ component. The $C$ component, however, has disappeared. The lower record illustrates the reflex EMG activity in response to auditory stimulation when sitting, pushing both feet into the ground. A small short latency A component is visible. The $B$ and $C$ components are relatively unchanged, compared to sitting relaxed. The differences between the reflex EMG patterns shown in the middle and lower records occur despite comparable levels of background EMG activity. Each record consists of three superimposed single trials of unrectified EMG activity. The auditory stimulus, a $50 \mathrm{~ms}$ duration tone of $1000 \mathrm{~Hz}$ and $124 \mathrm{~dB}$, was delivered at the beginning of each trial. The total sweep duration of each trial was $200 \mathrm{~ms}$.

of a muscle was dependent on the postural set of that muscle. Thus following an auditory stimulus, the earliest latency (A) component was not recorded at rest, but was recorded when the muscle was posturally important. Conversely, the $\mathrm{C}$ component, which was well formed at rest, was greatly diminished in size or absent when standing or crouching on all fours. The change in the EMG pattern of the reflex response to sound with postural activity was striking in cases 4 and 5 , and not simply the effect of increased background EMG activity.

Similarities between the various EMG components recorded in cases 4 and 5 suggest that a common efferent system was responsible for each component of the reflex response to auditory stimulation. In particular, the difference in latency to onset of reflex EMG activity between the biceps and tibialis anterior for each of the three EMG components was relatively long compared with the difference in latency between the same two muscles recorded following magnetic stimulation of the motor cortex (see table 1). This suggests that each of the three components of reflex EMG activity was subserved by a moderately slowly conducting spinal efferent pathway. Another point of similarity between the three EMG components was the disproportionately long relative latencies of the three components in the intrinsic hand and foot muscles in case 4, even when allowance was made for slow conduction in spinal efferent pathways.

WHAT IS THE BASIS FOR POSTURALLY INDUCED CHANGES IN THE PATTERN OF EMG ACTIVITY IN THE AUDITORY STARTLE REFLEX?

Animal experiments, ${ }^{7-9}$ and electrophysiological evidence in humans ${ }^{1}$ indicate that the physiological startle reflex originates in the lower brainstem. Acquired startle disease in humans is frequently associated with brainstem pathology, ${ }^{210}$ and the pattern of muscle recruitment in the abnormal startle response in hyperekplexia is very similar to that recorded in the physiological startle response. ${ }^{12}$ These observations suggest that the physiological startle reflex, and the pathological startle reflex in hyperekplexia represent physiological and pathological activity in the same brainstem efferent system. ${ }^{2}$ The spinal projections of this system are relatively slowly conducting, ${ }^{12}$ and bear some similarities to the relatively slowly conducting pathways subserving the efferent

The latencies to onset of the $A, B$ and $C$ components of the reflex EMG response to auditory stimulation in cases 4 and 5

\begin{tabular}{|c|c|c|c|c|c|c|c|c|c|c|}
\hline \multirow[b]{3}{*}{ Latency (MS) } & \multicolumn{10}{|l|}{ Case 4} \\
\hline & \multicolumn{3}{|l|}{$A$} & \multicolumn{3}{|l|}{$B$} & \multicolumn{3}{|l|}{$C$} & \multirow{2}{*}{$\begin{array}{l}\text { Cortical } \\
\text { stimulation }\end{array}$} \\
\hline & Median & Range & $N$ & Median & Range & $N$ & Median & Range & $N$ & \\
\hline \multirow[t]{4}{*}{$\begin{array}{l}\text { Biceps } \\
\text { FDI } \\
\text { FDI-Biceps } \\
\text { Lumbar paraspinals } \\
\text { TA } \\
\text { TA-Biceps } \\
\text { AHB } \\
\text { AHB-TA }\end{array}$} & $\begin{array}{l}31 \\
\text { N/A } \\
\text { N/A } \\
38 \\
57 \\
26 \\
70 \\
13\end{array}$ & $\begin{array}{l}30-35 \\
33-42 \\
54-61 \\
66-77\end{array}$ & $\begin{array}{l}7 \\
6\end{array}$ & $\begin{array}{r}57 \\
91 \\
34 \\
63 \\
84 \\
27 \\
102 \\
18\end{array}$ & $\begin{array}{l}54-69 \\
79-101 \\
\\
58-72 \\
79-93 \\
97-105\end{array}$ & $\begin{array}{r}10 \\
10 \\
8\end{array}$ & $\begin{array}{r}102 \\
123 \\
21 \\
106 \\
130 \\
28 \\
145 \\
15\end{array}$ & $\begin{array}{r}97-116 \\
113-134 \\
\\
101-113 \\
127-136 \\
132-155\end{array}$ & $\begin{array}{r}10 \\
10 \\
10 \\
7 \\
7\end{array}$ & $\begin{array}{l}11 \\
24 \\
13 \\
\mathrm{~N} / \mathrm{A} \\
31 \\
20 \\
42 \\
11\end{array}$ \\
\hline & \multicolumn{10}{|l|}{ Case 5} \\
\hline & \multicolumn{3}{|l|}{$A$} & \multicolumn{3}{|l|}{$B$} & \multicolumn{3}{|l|}{$C$} & \multirow{2}{*}{$\begin{array}{l}\text { Cortical } \\
\text { stimulation }\end{array}$} \\
\hline & Median & Range & $N$ & Median & Range & $N$ & Median & Range & $N$ & \\
\hline $\begin{array}{l}\text { Biceps } \\
\text { Rectus Abdominis } \\
\text { TA } \\
\text { TA-Biceps }\end{array}$ & $\begin{array}{l}36 \\
45 \\
60 \\
24\end{array}$ & $\begin{array}{l}31-42 \\
43-47 \\
46-67\end{array}$ & $\begin{array}{l}5 \\
5 \\
6\end{array}$ & $\begin{array}{l}52 \\
65 \\
78 \\
26\end{array}$ & $\begin{array}{l}46-61 \\
50-67 \\
72-79\end{array}$ & $\begin{array}{l}6 \\
8 \\
6\end{array}$ & $\begin{array}{l}\text { N/A } \\
\text { N/A } \\
114 \\
\text { N/A }\end{array}$ & $112-117$ & 4 & $\begin{array}{l}11 \\
N / A \\
25 \\
14\end{array}$ \\
\hline
\end{tabular}

The median latencies to onset of EMG activity in the A, B and C components elicited by auditory stimulation (a $50 \mathrm{~ms}$ duration tone of $1 \mathrm{Kz}$ and $124 \mathrm{db}$ ) in cases 4 and 5 . Median latencies and the differences between these latencies in representative pairs of in each case. Abbreviations: first dorsal interosseous (FDI), tibialis anterior (TA), abductor hallucis brevis (AHB), not available (N/A). 
limb of the spino-bulbo-spinal reflex in animals. ${ }^{11}$ The latter pathways form the basis of the audio-spinal reflex, believed to underlie the auditory startle reflex in animals. ${ }^{8}$

In this investigation we have shown that both the normal and abnormal auditory startle responses have complex EMG patterns dependent on the postural set of the limbs. Other reflex patterns may also be dependent on the postural set of a limb or muscle. ${ }^{12-16}$

The mechanism by which the EMG pattern of the pathological and physiological auditory startle response changes with posture is unclear. However, it may be relevant that among normal subjects and in patients with a pathologically exaggerated startle reflex there were three different latencies of response under different conditions. The longest latency to onset of reflex EMG activity in the tibialis anterior, about $120 \mathrm{~ms}$, was recorded in normal subjects when sitting relaxed. When normal subjects were standing the latency to onset of reflex EMG activity in the tibialis anterior was shorter, about $85 \mathrm{~ms}$, and similar to that recorded in hyperekplexic patients when sitting relaxed. The shortest latency to onset of reflex EMG activity, about $60 \mathrm{~ms}$, was recorded in the tibialis anterior when some hyperekplexic patients stood (cases 3, 4 and 5).

The possibility that the startle response in the limbs may, under different circumstances, have three preferred latencies was strengthened by the finding of three discrete EMG components of similar latency (about 60, 80 and $120 \mathrm{~ms}$ for the A, B and C EMG components in the tibialis anterior) in cases 4 and 5 . In these two patients the similarities between these three EMG components suggested that a common efferent system was responsible for each component of the reflex response to auditory stimulation. In particular, each component of the pathological auditory startle response utilised a moderately slowly conducting spinal efferent pathway, and was therefore consistent with activity in the efferent limb of the spino-bulbo-spinal reflex arc. Assuming, on this evidence, that a single efferent system is responsible for EMG components of differing latency, one possible explanation for differences in bsolute latency in the normal and exaggerated startle reflexes is that the startle response is the result of up to three basic waves of activity in bulbo-spinal efferent pathways. Postural and other influences may then operate independently on each wave of efferent activity. In the resting normal subject only the long latency component of the startle response is recorded in the lower limbs. When the normal subject stands, postural influences allow expression of the middle latency component. In many patients with hyperekplexia, disinhibition or facilitation of the startle response allows expression of the middle latency com- ponent when sitting relaxed. The earliest component of startle activity is most readily recorded in the lower limbs when postural influences combine with pathological disinhibition or facilitation in the standing hyperekplexic patient. The net effect is a shortening of the absolute latency of the reflex response to sound on standing in both normal subjects and patients with hyperekplexia.

The structures which mediate postural influences on activity in the bulbo-spinal efferent pathways in the startle response are unknown. Nor can these be the only influences on the activity in these pathways, as some normal subjects and some patients with exaggerated startle reflexes fail to adapt to changes in posture.

In conclusion, both the physiological and pathological auditory startle reflex have a complex EMG pattern dependent on the postural set of the limbs. This supports previous arguments ${ }^{8}$ suggesting a common origin for the normal and abnormal startle responses. More importantly, however, these results indicate that the auditory startle response is not immutable. Its physiological importance may then lie in rapidly accomplishing a defensive stance with maximum postural stability.

1 Brown P, Rothwell JC, Thompson PD, Britton TC, Day $B L$, Marsden $C D$. New observations on the normal auditory startle reflex in man. Brain (in press)

2 Brown P, Rothwell JC, Thompson PD, Britton TC, Day BL, Marsden CD. Observations on hyperekplexia and its relationship to the normal startle reflex. Brain (in press).

3 Landis C, Hunt WA. The startle pattern. New York: Farra and Rinehart, 1939.

4 Andermann F, Keene DL, Andermann E, Quesney LF. Startle disease or hyperekplexia: further delineation of the syndrome. Brain 1980;103:985-97.

5 Rossignol S. Startle responses recorded in the leg of man. Electroenceph clin Neurophysiol 1975;39:389-97.

6 Leigh PN, Rothwell JC, Traub M, Marsden CD. A patient with reflex myoclonus and muscle rigidity: "jerking stiffman syndrome." J Neurol Neurosurg Psychiatry man syndrome."

7 Szabo I, Hazafi K. Elicitability of the acoustic startle reaction after brainstem lesions. Acta Physiol Acad Sci Hung $1965 ; 27: 155-65$.

8 Wright CG, Barnes CD. Audio-spinal reflex responses in decerebrate and chloralose anaesthetised cats. Brain Res 1972;36:307-31.

9 Davis M, Gendelman DS, Tischler MD, Gendelman PM. Primary acoustic startle circuit: lesion and stimulation studies. J Neurosci 1982;2:791-805.

10 Fenzi F, Boniovanni G, Fincati E, Pampanin M, Tomelleri G, Rizzuto N. Anatomical and clinical study of a case of subacute encephalomyelitis with hyperekplexia drome. Ital J Neurol Sci 1988;9:505-8.

11 Shimamura M, Livingston RB. Longitudinal conduction systems serving spinal and brainstem coordination. $J$ systems serving spinal and
Neurophysiol 1963;26:258-72.

12 Kugelberg E, Eklund K, Grimby L. An electromyographic study of the nociceptive reflexes of the lower limb. Mechanism of the plantar response. Brain 1960;83 $394-410$.

13 Nashner LM. Adapting reflexes controlling the human posture. Exp Brain Res 1976;26:59-72.

14 Marsden CD, Merton PA, Morton HB. Human postural responses. Brain 1981;104:513-34.

15 Dieterich M, Brandt TH, Fries W. Otolith function in man: results from a case of otolith Tullio phenomenon. Brain 1989;112:1377-92.

16 Nardone A, Giordano A, Corra T, Schieppati M. Responses of leg muscles in humans displaced while standing: effects of types of pertubation and of postural set. Brain 